\title{
Dextromethorphan Abuse Among Opioid-Dependent Patients
}

\author{
Laura G. Windhab, * Salome Gastberger, MD, † Lea M. Hulka, MD,† Markus R. Baumgartner, MD, $\neq$ \\ Michael Soyka, MD, § Thomas J. Müller, MD, * Erich Seifritz, MD, // and Jochen Mutschler, MD*
}

Background: Among opioid-dependent patients on maintenance therapy, concomitant drug abuse is a serious problem. Dextromethorphan, an over-the-counter antitussive agent that can be purchased without prescription, is a drug with a high potential for misuse, especially when consumed in high doses.

The objective of this study was to investigate possible abuse of dextromethorphan among substituted opioid-dependent patients and comparison of subjective and objective findings.

Due to its ability to increase serotonin levels, opioid-dependent patients may be particularly susceptible to dextromethorphan misuse. Dextromethorphan misuse may have side effects, including psychiatric symptoms and serotonin syndrome, and may induce assault, suicide, or homicide.

Methods: A total of 104 opioid-dependent patients in maintenance treatment were included in this cross-sectional study conducted in the outpatient department of the Psychiatric Hospital, University of Zurich. Study participants were divided into 2 groups based on laboratory results: dextromethorphan abusers $(\mathrm{n}=12)$ and nonabusers $(\mathrm{n}=92)$. The objective use and concentrations of dextromethorphan was detected using 3-month hair toxicology analysis.

Statistical analysis was performed by using $\chi^{2}$ test, Student $t$ test, Mann-Whitney $U$ test, and Barnard exact test.

Results: Dextromethorphan was abused by 12 (11.5\%) patients, 11 (91.7\%) of whom did not report concomitant abuse of dextromethorphan but were identified through hair analysis. In general, there were significant differences among patients abusing dextromethorphan compared with nondextromethorphan consumers in terms of trauma due to sexual maltreatment/violence, multiple traumas, or harmful use of hallucinogenic drugs.

Conclusions: Further studies are necessary to examine dextromethorphan and its impact on patients with psychiatric comorbidities and psychiatric medication. According to literature, there is a significant drug interaction risk due to the impact of dextromethorphan misuse on serotonin syndrome and psychiatric symptoms. ${ }^{1-3}$ We recommend active inquiry into and testing for concomitant drug abuse among substituted opioid-dependent patients to reduce the risk of drug interactions and side effects in this especially vulnerable group of patients.

Key Words: dextromethorphan, opioid dependency, abuse

(Clin Neuropharm 2020;43: 127-133)

$\mathrm{D}$ extromethorphan, an $N$-methyl-D-aspartate (NMDA) antagonist and sigma-1 receptor agonist, is an antitussive agent that

*Psychiatric Hospital Meiringen, Meiringen; $†$ Department of Psychiatry, Center for Addictive Disorders, Psychotherapy and Psychosomatics, Psychiatric Hospital, $\ddagger$ Zurich Institute of Forensic Medicine, Center for Forensic Hair Analysis, University of Zurich, Zürich, Switzerland; §Psychosomatik, Bernau; |Psychiatric Hospital University of Munich, Munich, Germany; and \|Department of Psychiatry, Psychotherapy and Psychosomatics, Psychiatric Hospital, University of Zurich, Zürich, Switzerland.

Address correspondence and reprint requests to Laura G. Windhab,

Privatklinik Meiringen Postfach 6123860 Meiringen, Switzerland;

E-mail: Laura.Windhab@gmx.ch

Conflicts of Interest and Source of Funding: The authors have no conflicts of interest to declare.

Copyright (C) 2020 Wolters Kluwer Health, Inc. All rights reserved.

DOI: $10.1097 / \mathrm{WNF} .0000000000000403$ has been available as an over-the-counter medication in pharmacies without a prescription since 1954 . There has been an alarming increase in reports of its abuse cases (7- to 10-fold between 1999 and 2004), ${ }^{4}$ mainly among young people taking dextromethorphan as a psychoactive drug (tripled from 2000 to 2006 and plateaued from 2006 to 2015$)^{5}$

However, current data are incomplete regarding the extent of the problem and its significance in addiction medicine. ${ }^{6}$

Due to its NMDA receptor agonism and sigma-1 receptor antagonism, the pharmacodynamic effects of dextromethorphan are similar to lysergic acid diethylamide, psilocybin or ketamine., Consequently, dextromethorphan is a member of the group of substances with psychoactive (dissociative) effects. As its chemical structure is similar to that of codeine, dextromethorphan was classified as a synthetic opioid until recently. Because the sigma-1 receptor is no longer considered an opioid receptor, nowadays, dextromethorphan is accordingly no longer classified as an opioid. ${ }^{7,9,10}$ The antitussive effect is likely mediated by both NMDA and sigma-1 receptors. ${ }^{11}$ Gamma-aminobutyric acid (GABA)-ergic/ antiglutamatergic mechanisms play an important role in the etiopathogenesis of addictive behavior and in the development of dependency. ${ }^{12}$ Occasional substance use behavior often develops over time into uncontrolled, rigid, compulsive-repetitive conduct, and the development of tolerance is accompanied by autonomic withdrawal symptoms. ${ }^{13}$ In addition, many addictive substances reduce GABAergic neurotransmission and reinforce glutamatergic neurotransmission (glutamatergic hyperexcitability), with excitatory transmission provided by NMDA receptors (glutamate receptors). ${ }^{14}$ Soyka and colleagues showed that the NMDA antagonist dextromethorphan, acting as a glutamate antagonist, shows addictive potential due to the development of psychotropic effects analogous to those of alcohol. ${ }^{15}$

Taken at low doses, dextromethorphan rarely shows side effects, although itching, vertigo, fatigue, vomiting, and nausea are occasionally observed. ${ }^{16}$ Taken together with monoamine oxidase inhibitors, the antihistamine terfenadine or selective serotonin reuptake inhibitors, dextromethorphan has been linked to potentially life-threatening interactions, such as serotonin syndrome. ${ }^{16-18}$

Dextromethorphan is predominantly metabolized in the liver by cytochrome $\mathrm{P}_{450} 2 \mathrm{D} 6$ (CYP2D6). Around $1 \%$ to $3 \%$ of the German population are ultrarapid metabolizers, showing a high CYP2D6 activity level, whereas $7 \%$ to $10 \%$ are poor metabolizers who show no CYP2D6 activity. ${ }^{19}$ Many individuals, therefore, have an extended plasma half-life of dextromethorphan that can lead to psychoactive effects even at therapeutic doses.

Concomitant drug abuse is a serious issue, especially among opioid-dependent patients in replacement therapy. ${ }^{1}$ Clinical and epidemiological studies show that substance use disorders and psychiatric disorders are highly comorbid, ${ }^{2}$ and psychiatric disorders are often accompanied by diverse medications, such as selective serotonin reuptake inhibitors and others. Due to drug interactions, substituted patients who use dextromethorphan are, therefore, exposed to a higher risk of developing (additional) psychiatric symptoms or serotonin syndrome, because 
high doses of dextromethorphan can result in hallucinations and mania. ${ }^{3}$ Dextromethorphan use may also result in homicide, suicide, or assault, as reported in 5 cases. $^{3}$

Dextromethorphan is nowadays marketed as a constituent of a large number of generic drugs. Between 2007 and 2008, sales of 1 commercial preparation rose by $32.9 \%$ (measured in defined daily dose) for unknown reasons. ${ }^{20}$

However, to date, there are no detailed epidemiological data on dextromethorphan abuse as a psychoactive substance, although at least 5 cases of confirmed dextromethorphan dependency have been described in the literature so far. ${ }^{21}$

In this study, we investigated the proportion of study participants who used dextromethorphan in addition to their replacement therapy. Because of their concomitant drug abuse, the participants illustrate a vulnerable group of patients due to drug interactions and related side effects. We, therefore, checked for specificities in their appearances and related behavioral patterns. Subjective patient statements were first collected with the help of questionnaires, followed by a 3-month hair toxicology analysis that objectively determined the ingestion and concentrations of dextromethorphan.

\section{MATERIALS AND METHODS}

\section{Subjects}

Hair samples were originally analyzed in the context of a study conducted in an outpatient setting at the Centre for Addictive Disorders of the Psychiatric Hospital, University of Zurich in 2014. ${ }^{22,23}$ From that study, we analyzed in further detail a range of substances

TABLE 1. Patient Characteristics

\begin{tabular}{|c|c|c|c|c|}
\hline Patient Characteristics & $\operatorname{DXM}(n=12)$ & No DXM $(n=92)$ & Total $(N=104)$ & $\boldsymbol{P}$ \\
\hline Sex, n (\%) & & & & $0.221 *$ \\
\hline Male & $7(58.3)$ & $69(75)$ & $76(73.1)$ & $0.254 \dagger$ \\
\hline Female & $5(41.7)$ & $23(25)$ & $28(26.9)$ & \\
\hline Age $($ mean $\pm \mathrm{SD})$ & $37.6 \pm 6.9$ & $41.63 \pm 8.7$ & $41.16 \pm 8.6$ & $0.125 \ddagger$ \\
\hline School, n (\%) & & & & $0.466^{*}$ \\
\hline None & $1(8.3)$ & $3(3.3)$ & $4(3.8)$ & \\
\hline Middle school & $4(33.3)$ & $30(32.6)$ & $34(32.7)$ & \\
\hline High school & $7(58.3)$ & $46(50)$ & $53(51)$ & \\
\hline Academic high school & $0(0)$ & $13(14.1)$ & $13(12.5)$ & \\
\hline Education, n (\%) & & & & $0.407 *$ \\
\hline None & $5(41.7)$ & $20(21.7)$ & $25(24)$ & \\
\hline Apprenticeship & $7(58.3)$ & $65(70.7)$ & $72(69.2)$ & \\
\hline University of applied science & $0(0)$ & $5(5.4)$ & $5(5.4)$ & \\
\hline University & $0(0)$ & $2(2.2)$ & $2(2.2)$ & \\
\hline Employed, n (\%) & & & & $0.344 *$ \\
\hline Full-time & $0(0)$ & $7(7.6)$ & $7(6.7)$ & \\
\hline Part-time & $0(0)$ & $7(7.6)$ & $7(6.7)$ & \\
\hline Unemployed & $0(0)$ & $10(10.9)$ & $10(9.6)$ & \\
\hline Disability & $7(58.3)$ & $51(55.4)$ & $58(55.8)$ & \\
\hline Student & $0(0)$ & $1(1.1)$ & $1(1)$ & \\
\hline Certified sick & $1(8.3)$ & $4(4.3)$ & $5(4.8)$ & \\
\hline Other & $4(33.3)$ & $10(10.9)$ & $14(13.5)$ & \\
\hline Not classified & $0(0)$ & $2(2.2)$ & $2(1.9)$ & \\
\hline No. psychiatric comorbidities (mean \pm SD) & $1.58 \pm 0.99$ & $1.61 \pm 1.16$ & $1.61 \pm 1.14$ & $0.942 \ddagger$ \\
\hline No. clinical treatments (mean $\pm \mathrm{SD})$ & $5.5 \pm 7.66$ & $6.52 \pm 7.61$ & $6.40 \pm 7.58$ & $0.662 \ddagger$ \\
\hline $\mathrm{BDI}($ mean $\pm \mathrm{SD})$ & $18.08 \pm 8.21$ & $16.15 \pm 9.19$ & $16.38 \pm 9.06$ & $0.491 \ddagger$ \\
\hline HIV, n $(\%)$ & & & & $0.971 *$ \\
\hline Yes & $1(8.3)$ & $6(6.52)$ & $7(6.73)$ & $0.962 \dagger$ \\
\hline No & $9(75)$ & $71(77.17)$ & $80(76.92)$ & \\
\hline Unknown & $2(16.66)$ & $15(16.3)$ & $17(16.35)$ & \\
\hline Hepatitis C, n (\%) & & & & $0.204 *$ \\
\hline Yes & $7(58.33)$ & $36(39.13)$ & $43(41.35)$ & $0.217 \dagger$ \\
\hline No & $5(41.66)$ & $56(60.87)$ & $61(58.65)$ & \\
\hline Serotonergic medication (fluoxetin, citalopram, trimipramin) & & & & $0.645^{*}$ \\
\hline Yes & $3(25)$ & $29(31.52)$ & $32(30.77)$ & $0.836 \dagger$ \\
\hline No & $9(75)$ & $63(68.48)$ & $72(69.23)$ & \\
\hline
\end{tabular}

*Pearson $x^{2}$ test.

$\dagger$ Barnard exact test.

† Student $t$ test. 
TABLE 2. Subjective and Objective Findings

\begin{tabular}{|c|c|c|c|c|}
\hline Group Characteristics & DXM $(n=12)$ & No DXM $(n=92)$ & Total $(N=104)$ & $\boldsymbol{P}$ \\
\hline Dextromethorphan subjective, $\mathrm{n}(\%)$ & & & & $0.005^{*}$ \\
\hline Yes & $1(8.33)$ & $0(0)$ & $1(0.96)$ & $0.047 \dagger$ \\
\hline No & $11(91.67)$ & $92(100)$ & $103(99.04)$ & \\
\hline Dextromethorphan objective, n (\%) & & & & $0.000 *$ \\
\hline Yes & $12(100)$ & $0(0)$ & $12(11.54)$ & $0.000 \dagger$ \\
\hline No & $0(0)$ & $92(100)$ & $92(88.46)$ & \\
\hline Cocaine subjective, n (\%) & & & & $0.277^{*}$ \\
\hline Yes & $9(75)$ & $54(58.69)$ & $63(60.58)$ & $0.318 \dagger$ \\
\hline No & $3(25)$ & $38(4.30)$ & $41(39.42)$ & \\
\hline Cocaine objective, $\mathrm{n}(\%)$ & & & & $0.277^{*}$ \\
\hline Yes & $9(75)$ & $54(58.69)$ & $63(60.58)$ & $0.318 \dagger$ \\
\hline No & $3(75)$ & $38(4.30)$ & $41(39.42)$ & \\
\hline Heroine subjective, n (\%) & & & & $0.751 *$ \\
\hline Yes & $5(41.67)$ & $34(36.96)$ & $39(37.5)$ & $0.943 \dagger$ \\
\hline No & $7(58.33)$ & $58(63.04)$ & $65(62.5)$ & \\
\hline Heroine objective, $\mathrm{n}(\%)$ & & & & $0.646^{*}$ \\
\hline Yes & $4(33.33)$ & $37(40.22)$ & $41(39.42)$ & $0.836 \dagger$ \\
\hline No & $8(66.67)$ & $55(59.78)$ & $63(60.58)$ & \\
\hline Methylphenidate subjective, $\mathrm{n}(\%)$ & & & & $0.069 *$ \\
\hline Yes & $6(50)$ & $23(25)$ & $29(27.88)$ & $0.072 \dagger$ \\
\hline No & $6(50)$ & $69(75)$ & $75(72.12)$ & \\
\hline Methylphenidate objective, n (\%) & & & & $0.776^{*}$ \\
\hline Yes & $6(50)$ & $50(54.35)$ & $56(53.85)$ & $0.943 \dagger$ \\
\hline No & $6(50)$ & $42(45.65)$ & $48(46.15)$ & \\
\hline
\end{tabular}

*Pearson $x^{2}$ test.

$\dagger$ Barnard exact test.

in 104 patients, including 76 male and 28 female participants, in opioid substitution therapy.

All 104 patients were included in this cross-sectional study, including 12 with comorbid dextromethorphan use and 92 patients with no dextromethorphan use.

The inclusion criteria were a diagnosis of opioid dependence according to Diagnostic and Statistical Manual of Mental Disorders IV criteria and participation in opioid substitution therapy at the time of the study. Insufficient command of the German language or younger than 18 years and older than 65 years were exclusion criteria. Neither prescribed comedication nor psychiatric comorbidities were excluded.

All participants gave written informed consent before participation in the study and received a payment of 5 Swiss Francs. The ethics committee of the canton of Zurich approved the study protocol.

\section{Study Design}

The participants were divided into 2 groups on the basis of laboratory hair toxicology analysis of dextromethorphan abuse ( $\mathrm{n}=12$; including 7 male and 5 female participants) or no dextromethorphan consumption $(\mathrm{n}=92$; including 69 male and 23 female participants).

The Institute of Forensic Medicine at the University of Zurich evaluated drug use based on a 3-month hair toxicology analysis that quantifies the concentration of the substances or their metabolites. This analysis also revealed the fraction of hair samples in which dextromethorphan was represented.

With the help of electronic medical records, data were collected on psychiatric diagnoses based on ICD-10. Demographic data, such as sex, age, years and type of education, employment status, as well as the number of clinical treatments, were obtained by self-reporting via questionnaires.

Craving was assessed using the visual analog scale, ranging from 1 to 100 (Rosenberg, 2009). ${ }^{24}$ Depression and anxiety were analyzed with help of the Beck Depression Inventory (BDI) ${ }^{25}$ and the State-Trait Anxiety Inventory ${ }^{26}$ (Beck et al., 1961; Spielberger et al., 1983).

\section{Study Assessment}

The outcome of the study was a comparison of the subjective reporting of dextromethorphan abuse to objective hair concentrations of dextromethorphan found in substituted opioid-dependent patients. Concentrations of dextromethorphan in hair samples were tested and quantified by 3-month hair toxicology analysis using liquid chromatography-tandem mass spectrometry. Concentrations lower than $60 \mathrm{pg} / \mathrm{mg}$ were considered negligible because they do not represent abusive consumption of dextromethorphan, which led to 3 of 15 patients being regarded as unremarkable dextromethorphan users. ${ }^{27}$ The remaining 12 patients were classified as dextromethorphan abusers due to a concentration greater than $60 \mathrm{pg} / \mathrm{mg}$ dextromethorphan in their hair samples.

\section{Hair Analysis}

A 3-month hair toxicology analysis, carried out at the Institute of Forensic Medicine at the University of Zurich, was used for assessing abusive dextromethorphan consumption. Using head hair, the proximal $3-\mathrm{cm}$ section of the collected head hair locks was investigated. Whenever little or no, or only heavily bleached 
TABLE 3. Psychiatric Comorbidity: Traumas

\begin{tabular}{|c|c|c|c|c|}
\hline Patient Characteristics & $\operatorname{DXM}(n=12)$ & No DXM $(n=92)$ & Total $(N=104)$ & $\boldsymbol{P}$ \\
\hline Simple PTSD, n (\%) & & & & $0.685^{*}$ \\
\hline Yes & $1(8.33)$ & $5(5.43)$ & $6(5.77)$ & $0.881 \dagger$ \\
\hline No & $11(91.67)$ & 87 (94.57) & $98(94.23)$ & \\
\hline Complex PTSD, n (\%) & & & & $0.268^{*}$ \\
\hline Yes & $3(25)$ & $12(13.04)$ & $15(14.42)$ & $0.317 \dagger$ \\
\hline No & $9(75)$ & $80(86.96)$ & $89(85.58)$ & \\
\hline Trauma neglect, n (\%) & & & & $0.788^{*}$ \\
\hline Yes & $1(8.33)$ & $10(10.87)$ & $11(10.58)$ & $0.963 \dagger$ \\
\hline No & $11(91.67)$ & $82(89.13)$ & $93(89.42)$ & \\
\hline Trauma sexual abuse, n (\%) & & & & $0.39 *$ \\
\hline Yes & $1(8.33)$ & $3(3.26)$ & $4(3.85)$ & $0.49 \dagger$ \\
\hline No & $11(91.67)$ & $89(96.74)$ & $100(96.15)$ & \\
\hline Trauma sexual maltreatment/violence, n (\%) & & & & $0.014 *$ \\
\hline Yes & $2(16.67)$ & $2(2.17)$ & $4(3.85)$ & $0.0485 \dagger$ \\
\hline No & $10(83.33)$ & $90(97.82)$ & $100(96.15)$ & \\
\hline Trauma violence, $\mathrm{n}(\%)$ & & & & $0.294 *$ \\
\hline Yes & $2(16.67)$ & $7(7.61)$ & $9(8.65)$ & $0.369 \dagger$ \\
\hline No & $10(83.33)$ & $85(92.39)$ & $95(91.35)$ & \\
\hline Multiple traumas, n (\%) & & & & $0.032 *$ \\
\hline Yes & $3(25)$ & $6(6.52)$ & $9(8.65)$ & $0.0485 \dagger$ \\
\hline No & $9(75)$ & $86(93.48)$ & $95(91.35)$ & \\
\hline $\begin{array}{l}\text { *Pearson } \chi^{2} \text { test. } \\
\dagger \text { Barnard exact test. } \\
\text { PTSD, posttraumatic stress disorder. }\end{array}$ & & & & \\
\hline
\end{tabular}

hair was available, body hair was used. Assuming an average growth rate of $1 \mathrm{~cm} /$ month, the hair segment represents the consumption behavior of the last 3 months before collection. ${ }^{23}$ The analytical limits of the method enable the detection of very low concentration in the hair samples. However, strong cosmetic treatments like bleaching can degrade the incorporated substances. ${ }^{28}$ Hair samples were analyzed according to Rust et al. ${ }^{29}$ In brief, the hair sample was washed in water, acetone, and hexane for 2 minutes each. Then dried at room temperature, cut into small snippets, and extracted in methanol (extraction step 1) and a mixture of methanol and ammonium formate buffer (extraction step 2) for 90 minutes each. After each extraction step, the organic layers were separated by centrifugation and dried under a stream of nitrogen at $36^{\circ} \mathrm{C}$. Finally, the residues were combined, redissolved in methanol and aqueous ammonium format buffer solution, and then the samples were analyzed with liquid chromatography-tandem mass spectrometry. ${ }^{23}$

\section{Statistical Analysis}

\section{General Aspects of Applied Statistical Methods}

Data analysis was performed using Excel for Mac, version 15.29. The $\chi^{2}$ test was used for categorical variables and Student $t$ test for continuous variables. Differences in baseline characteristics between the 2 groups were compared. The Mann-Whitney $U$ test was used to identify nonparametric variables to compare quantitative dextromethorphan use between the 2 groups. ${ }^{30}$

The results of the hair analysis were recoded to count the percentages of study participants with dextromethorphan use. All values of 0 were classified as no, and all values greater than 0 were classified as yes. With the help of the $\chi^{2}$ test the 2 groups were compared, and the null hypothesis ( $\mathrm{X}$ and $\mathrm{Y}$ characteristics are independent) confirmed or rejected.
Accordingly, we considered $P$ values less than 0.05 as statistically significant and conditional for rejecting the null hypothesis and thus confirming a dependency of the $\mathrm{X}$ and $\mathrm{Y}$ characteristics.

\section{Weakness of the $\chi^{2}$ independence test}

The $\chi^{2}$ goodness of fit test is appropriate when the following conditions are met: (1) the sampling method is simple random sampling, (2) the variable under study is categorical, and (3) the expected value of the number of sample observations for each variable is at least 5 .

The data in this study only partially fulfill condition 3 because although the group of comorbid dextromethorphan abuse patients counted 12 subjects, certain subgroups with side effects such as psychiatric symptoms, serotonin syndrome, assault, suicide, or homicide often consisted of fewer than 5 subjects.

Accordingly, we also used exact statistical methods of relevance for smaller sample numbers.

\section{Exact Statistical Tests for Independence}

There are 2 fundamentally different exact tests for comparing the equality of 2 binomial probabilities - Fisher exact test (Fisher, 1925), ${ }^{31}$ and Barnard exact test (Barnard, 1945). ${ }^{32,33}$ Fisher exact test is the more popular of the 2 . The main difference between the Fisher and Barnard tests is the manner in which they eliminate a nuisance parameter $\pi$ from a test statistic measuring how discrepant any Table $\mathrm{X}$ is relative to the type of table that would be expected under the null hypothesis, without sacrificing exactness.

To eliminate the unknown nuisance parameter $\pi$ in the $P$ value calculation, Barnard suggested calculating $\mathrm{p}(\pi)$ for all possible values of $\pi \in(0,1)$ and choosing the value, $\pi^{*}$, that maximizes $\mathrm{p}(\pi)$. This "Barnard $P$ value" $\mathrm{p}_{\mathrm{B}}$ is smaller than that generated by 
TABLE 4. ICD-10 Diagnosis

\begin{tabular}{|c|c|c|c|c|}
\hline Patient Characteristics & DXM $(n=12)$ & No DXM $(n=92)$ & Total $(N=104)$ & $\boldsymbol{P}$ \\
\hline F14.1 Use of cocaine, harmful use, n (\%) & & & & $1^{*}$ \\
\hline Yes & $3(25)$ & $23(25)$ & $26(25)$ & $1 \dagger$ \\
\hline No & $9(75)$ & $69(75)$ & $78(75)$ & \\
\hline F14.2 Use of cocaine, dependence syndrome, $\mathrm{n}(\%)$ & & & & $0.470 *$ \\
\hline Yes & $6(50)$ & $36(39.13)$ & $42(45.65)$ & $0.608 \dagger$ \\
\hline No & $6(50)$ & $56(60.87)$ & $62(59.62)$ & \\
\hline F15.1 Use of stimulants, harmful use, n (\%) & & & & $0.362 *$ \\
\hline Yes & $0(0)$ & $6(6.52)$ & $6(5.77)$ & $0.448 \dagger$ \\
\hline No & $12(100)$ & $86(93.48)$ & $98(94.23)$ & \\
\hline F15.2 Use of stimulants, dependence syndrome, n (\%) & & & & $0.382 *$ \\
\hline Yes & $1(8.33)$ & $17(18.48)$ & $18(17.31)$ & $0.476 \dagger$ \\
\hline No & $11(91.67)$ & $75(81.52)$ & $86(82.69)$ & \\
\hline F16.1 Use of hallucinogens, harmful use, n (\%) & & & & $0.005^{*}$ \\
\hline Yes & $1(8.33)$ & $0(0)$ & $1(0.96)$ & $0.047 \dagger$ \\
\hline No & $11(91.67)$ & $92(100)$ & $103(99.04)$ & \\
\hline F16.2 Use of hallucinogens, dependence syndrome, n (\%) & & & & $0.526^{*}$ \\
\hline Yes & $0(0)$ & $3(3.26)$ & $3(2.88)$ & $0.682 \dagger$ \\
\hline No & $12(100)$ & $89(96.74)$ & $101(97.12)$ & \\
\hline
\end{tabular}

*Pearson $\chi^{2}$ test.

$\dagger$ Barnard exact test.

Fisher exact test $\left(\mathrm{p}_{\mathrm{F}}\right)$, and thus suggests that Barnard test is more powerful than Fisher. This is a direct consequence of restricting Fisher test to $2 \times 2$ tables belonging to a conditional reference, rather than Barnard larger unconditional reference set and mirrors the much more discrete sample space for Fisher exact test than for Barnard exact test.

When finally comparing Fisher and Barnard exact tests, the loss of power due to the greater discreteness of the Fisher statistic is somewhat offset by the requirement that Barnard exact test must maximize over all possible $P$ values, by choice of the nuisance parameter $\pi$. For $2 \times 2$ tables, the loss of power due to discreteness dominates over the loss of power due to maximization, resulting in greater power for Barnard exact test. For details, see also Mehta and Hilton. ${ }^{34}$

Accordingly, we decided to apply Barnard exact test to prove or reject our null hypothesis of XY independency and compare the results to those of the $\chi^{2}$ test (see Tables $1-4$, for included $2 \times 2$ matrices).

\section{RESULTS}

Of the 104 study participants, 76 (73.1\%) were male and 28 $(26.9 \%)$ were female, with a mean $( \pm \mathrm{SD})$ age of 41.16 years ( \pm 8.6 years). Two groups were studied; dextromethorphan abusers $(\mathrm{n}=12)$ and nondextromethorphan consumers $(\mathrm{n}=92)$. Table 1 shows detailed patient characteristics. There were no significant differences between these 2 groups in terms of sex, type, and years of education, employment status, number of psychiatric comorbidities, BDI score, number of inpatient treatments, HIV status, hepatitis $\mathrm{C}$ or serotonergic medication.

Among the participants, 12 (11.5\%) patients were identified as dextromethorphan abusers through hair analysis. Of this group, $11(91.7 \%)$ patients failed to report abuse of dextromethorphan. Detailed patient statements are shown in Table 2 . For all 12 of the dextromethorphan abusers who additionally consumed cocaine, heroin, and methylphenidate, this additional abuse was reported.
Three (25\%) participants abusing dextromethorphan additionally took serotonergic medication, such as fluoxetine, citalopram, or trimipramin. Furthermore, patients were examined regarding psychiatric comorbidities, such as posttraumatic stress disorder and various types of traumas.

As shown in Table 3, we also identified a related statistically significant relationship between (a) trauma due to sexual maltreatment/ violence and (b) multiple traumas, and dextromethorphan abuse.

In addition, a similarly significant ( $5 \%$ level) dependency was identified for the harmful use of hallucinogens for 1 person within the group of dextromethorphan abusers, as illustrated in Table 4 and confirmed by both statistical tests.

\section{DISCUSSION}

Patients on maintenance therapy generally have multiple associated psychiatric comorbidities, ${ }^{29,35,36}$ are often treated with psychotropic drugs, and are frequently active drug abusers. ${ }^{37}$ This type of patient group is, therefore, more exposed to the risk of drug interactions that may then result in further psychiatric symptoms, serotonin syndrome, or even serious incidents, such as assaults, homicide, or suicide. ${ }^{2}$ With the help of clinical diagnoses, we attempted to determine whether dextromethorphan abusers show predictors or distinctive features. Regarding patient characteristics, such as sex, type and years of education, employment status, number of psychiatric comorbidities, BDI score and numbers of inpatient treatments, HIV status and hepatitis C or types of trauma, such as simple PTBS, complex PTBS, trauma through neglect, trauma through sexual abuse and trauma through violence, there were no statistically significant differences between dextromethorphan abusers and nondextromethorphan abusers. However, statistically significant differences were identified in relation to (1) trauma through sexual maltreatment/violence, (2) multiple traumas, and (3) the harmful use of hallucinogenic drugs among the dextromethorphan abuser group. To date, there are no indications in the literature 
for trauma due to sexual maltreatment/violence or multiple traumas as possible predictors of dextromethorphan misuse.

Finally, we were not able to explain the discrepancy between subjective statements and the proven abuse of dextromethorphan. This was especially puzzling because these patients were aware that admitting abuse would have no negative consequences. The concealment of dextromethorphan consumption among opioid abusers willing to admit concomitant cocaine, heroin and methylphenidate consumption remains to be explained. It is hypothesized that at the time of execution of the base study (2014), dextromethorphan was not identified by the members of the abusers group as a relevant drug impacting on their status like, for example, compared with heroin or cocaine, because dextromethorphan could be received by over-the-counter sales.

\section{CONCLUSION}

We recommend active inquiry and testing for concomitant dextromethorphan abuse among opioid-dependent patients to avoid drug interactions and side effects in this especially vulnerable group of patients. In this context, we also recommend further exploration of (a) trauma due to sexual maltreatment/violence and (b) multiple traumas as possible predictors of dextromethorphan misuse.

\section{ACKNOWLEDGMENT}

The authors thank all the investigators and patients who participated in this cross-sectional study.

Statement of Ethics: All participants provided written informed consent before admission to the study and were compensated with 5 Swiss Francs for their participation. The study protocol was approved by the local Ethics Committee of the canton of Zurich.

Study limitations: The size of the whole sample $(n=104)$ and the subsample of dextromethorphan abusers $(n=12)$ is rather small.

The expected value of the number of sample observations for each variable should be at least 5 for the $\chi^{2}$ test which is not always the case accordingly Exact statistical tests for independence had to be applied which has a remaining weakness in the estimate of the nuisance parameter $\pi$ by approximation.

Another test limitation relates to the fact that for the group of dextromethorphan abusers there was a discrepancy between subjective statements and the proven abuse of dextromethorphan (for 11 of 12). This means that the patients in the abuser group applied dextromethorphan as an antitussive agent due to its over-thecounter purchase and did not report its use as a drug.

The abovementioned limitations would have only shifted the ratio between objective and subjective identification results for the abusers group but had not impacted on the statistical evaluation of possibly correlated traumas.

The differentiation between use and abuse of dextromethorphan has been defined from the hair toxicology analysis as advised by the toxicological expert in charge.

Disclosure Statement: J.M. has received travel expenses and speaker fees from Lundbeck, Indivior, Jansen and Takeda. M.S. has worked as consultant and has received travel or research grants from Lundbeck, Indivior, and Mundipharma. M.S. has also worked as a consultant for Indivior and Camurus for the past 3 years.

T.M. has received speaker's fees from: AstraZeneca, Bristol-Myers Squibb, Janssen, Servier, Eli Lilly, Zeller Medical, Mepha and Sandoz. He is or has been a consultant to: Lundbeck, Otsuka, Sunovion, Bristol-Myers Squibb, AstraZeneca, Recordati and Janssen.

\section{REFERENCES}

1. Wagner E, Raabe F, Martin G, et al. Concomitant drug abuse of opioid dependent patients in maintenance treatment detected with a multi-target screening of oral fluid. Am J Addict 2018;27:407-412.
2. Kelly TM, Daley DC. Integrated treatment of substance use and psychiatric disorders. Soc Work Public Health 2013;28:388-406.

3. Logan BK, Yeakel JK, Goldfogel G, et al. Dextromethorphan abuse leading to aussalt, suicide, or homicide. J Forensic Sci 2012;57(5):1388-1394.

4. Bryner JK, Wang UK, Hui JW, et al. Dextromethorphan abuse in adolescence. Arch Pediatr Adolesc Med 2006;160(12):1217-1222.

5. Kerami S, Major JM, Calderon S, et al. Trends in dextromethorphan cough and cold products: 2000-2015 National Poison Data System intentional abuse exposure calls. Clin Toxicol 2018;56:656-663.

6. Mutschler J, Koopmann A, Grosshans M, et al. Dextromethorphan withdrawal and dependence syndrome. Dtsch Arztebl Int 2010;107(30): $537-540$

7. Miller SC. Dextromethorphan psychosis, dependence and physical withdrawal. Addict Biol 2005;10(4):325-327.

8. Romanelli F, Smith KM. Dextromethorphan abuse: clinical effects and management. J Am Pharm Assoc 2003 2009;49(2):e 20-e 25; quiz e26-7.

9. Chou YC, Liao JF, Chang WY, et al. Binding of dimemorfan to sigma-1-receptor and its anticonvulsant and locomotor effects in mice, compared with dextromethorphan and dextrophan. Brain Res 1999;821(2) 516-519.

10. Bolshakova AV, Kukanova EO, Gainullina AN, et al. Sigma-1 receptor as a potential pharmacological target for the treatment of neuropathology. St Petersburg Polytech Univ J Physics Math 2016;2(1):31-40.

11. Canning BJ. Central regulation of the cough reflex: therapeutic implications. Pulm Pharmacol Ther 2009;22(2):75-81.

12. Scofield MD, Heinsbroek JA, Gipson CD, et al. The nucleus accumbens: mechanisms of addiction across drug classes reflect the importance of glutamate homeostasis. Pharmacol Rev 2016;68(3):816-871.

13. Hodding GC, Jann M, Ackerman IP. Drug withdrawal syndromes - a literature review. West J Med 1980;133(5):383-391.

14. D'Souza MS. Glutamatergic transmission in drug reward: implications for drug addiction. Front Neurosci 2015;9:404.

15. Soyka M, Bondy B, Eisenburg B, et al. NMDA receptor challenge with dextromethorphan - subjective response, neuroendocrinological findings and possible clinical implications. J Neural Transm (Vienna) 2000;107(6): 701-714.

16. Bem J, Peck R. Dextromethorphan. An overview of safety issues. Drug Saf 1992;7(3):190-199.

17. Kintz $\mathrm{P}$, Mangin P. Toxicological findings in a death involving dextromethorphan and terfenadine. Am J Forensic Med Pathol 1992;13(4): 351-352.

18. Dy P, Arcega V, Ghali W, et al. Serotonin syndrome caused by drug to drug interaction between escitalopram and dextromethorphan. BMJ Case Rep 2017;2017:bcr-2017-bcr-221486.

19. Sachse C, Brockmöller J, Bauer S, et al. Cytochrome P450 2D6 variants in a Caucasian population: allele frequencies and phenotypic consequences. Am J Hum Genet 1997;60(2):284-295.

20. Lemmer B. Antitussiva. In: Schwabe U, Paffrath D, eds. Arzneiverordnungs-Report 2009. Berlin, Heidelberg, New York: Springer; 2009:419.

21. Logan BK, Goldfogel G, Hamilton R, et al. Five deaths resulting from abuse of dextromethorphan sold over the internet. J Anal Toxicol 2009; 33(2):99-103.

22. Mutschler J, Gastberger S, Baumgartner MR, et al. Pregabalin use among opioid-addicted patients in Switzerland. J Clin Psychiatry 2016;77(9): 1202-1203.

23. Gastberger S, Baumgartner MR, Soyka M, et al. Concomitant heroin and cocaine use among opioid-dependent patients during methadone, buprenorphine or morphine opioid agonist therapy. Eur Addict Res 2019; 25:207-212. 
24. Rosenberg H. Clinical and laboratory assessment of the subjective experience of drug craving. Clin Psychol Rev 2009;29(6):519-519.

25. Beck AT, Ward $\mathrm{CH}$, Mendelson $\mathrm{M}$, et al. An Inventory for Measuring Depression. Arch Gen Psychiatry 1961;4(6):561-571.

26. Spielberger CD. Manual for the State-Trait Anxiety Inventory (STAI). Palo Alto, CA: Consulting Psychologists Press; 1983.

27. Private communication with Head of Center for Forensic Hairanalytics Dr. Markus R. Baumgartner. (https://www.irm.uzh.ch/de/forschung/ fptforschung/team/C2.html).

28. Pötsch L, Skopp G. Stability of opiates in hair fibers after exposure to cosmetic treatment. Forensic Sci Int 1996;81(2-3):95-102.

29. Rust KY, Baumgartner MR, Meggiolaro N, et al. Detection and validated quantification of 21 benzodiazepines and 3 "z-drugs" in human hair by LC-MS/MS. Forensic Sci Int 2012;215(1-3):64-72.

30. Hartung J, Elpelt B, Klösener KH, eds. Statistik: Lehr- und Handbuch der angewandten Statistik. Walter de Gruyter GmbH \& Co KG; 2014.
31. Fisher RA, ed. Statistical Methods for Research Workers. Edinburgh: Oliver and Boyd; 1925.

32. Barnard GA. A new test for 2 x 2 tables. Nature 1945;156(3954):177.

33. Cardillo G. My Barnard: a very compact routine for Barnard's exact test on $2 \times 2$ matrix. 2019. Available from: http://www.mathworks.com/ matlabcentral/fileexchange/25760.

34. Mehta CR, Hilton JF. Exact power of conditional and unconditional tests: going beyond the 2x2 contingency table. Am Stat 1993;47(2):91-98.

35. Ahmad B, Mufti KA, Farooq S. Psychiatric comorbidity in substance abuse (opioids). J Pak Med Assoc 2001;51(5):183-186.

36. Langås AM, Malt UF, Opjordsmoen S. Comorbid mental disorders in substance use from a single catchment area - a clinical study. BMC Psychiatry 2011;11:25.

37. Swendsen J, Conway KP, Degenhardt L, et al. Mental disorders as risk factors for substance use, abuse and dependence: results from the 10-year follow-up of the National Comorbidity Survey. Addiction 2010;105(6):117-1128. 